\title{
Bright infrared quantum-dot light-emitting diodes through inter-dot spacing control
}

\author{
Liangfeng Sun ${ }^{1 / *}{ }^{\star}$, Joshua J. Choi ${ }^{12}$, David Stachnik', Adam C. Bartnik1, Byung-Ryool Hyun', \\ George G. Malliaras ${ }^{3 \dagger}$, Tobias Hanrath ${ }^{2}$ and Frank W. Wise ${ }^{1 \star}$
}

Infrared light-emitting diodes are currently fabricated from direct-gap semiconductors using epitaxy, which makes them expensive and difficult to integrate with other materials. Light-emitting diodes based on colloidal semiconductor quantum dots, on the other hand, can be solution-processed at low cost, and can be directly integrated with silicon'. However, so far, exciton dissociation and recombination have not been well controlled in these devices, and this has limited their performance ${ }^{2-8}$. Here, by tuning the distance between adjacent PbS quantum dots, we fabricate thin-film quantumdot light-emitting diodes that operate at infrared wavelengths with radiances $\left(6.4 \mathrm{~W} \mathrm{sr}^{-1} \mathrm{~m}^{-2}\right)$ eight times higher and external quantum efficiencies $(2.0 \%)$ two times higher than the highest values previously reported. The distance between adjacent dots is tuned over a range of $1.3 \mathrm{~nm}$ by varying the lengths of the linker molecules from three to eight $\mathrm{CH}_{2}$ groups, which allows us to achieve the optimum balance between charge injection and radiative exciton recombination. The electroluminescent powers of the best devices are comparable to those produced by commercial InGaAsP light-emitting diodes. By varying the size of the quantum dots, we can tune the emission wavelengths between 800 and 1,850 nm.

Colloidal quantum dots have been proposed for the development of low-temperature solution-processed quantum-dot devices, including next-generation photovoltaics, photodetectors and lightemitting diodes (LEDs) ${ }^{1-11}$. In particular, the development of high-power, efficient and low-cost infrared LEDs will further progress in applications such as night vision, optical communications and sensing. Early efforts to exploit quantum dots in LEDs were based on hybrid device structures in which the quantum dots were interfaced with conjugated polymers. Quantum dots with long capping ligands were either mixed with an organic host or directly sandwiched between organic carrier-transporting layers to form the LED structure ${ }^{4,5,8}$. The operating mechanism of such devices is based mainly on Förster transfer, in which exciton energy transfers from the organic host to the quantum dots by means of a dipole-dipole interaction. Owing to the long capping ligands and low carrier mobility of the organic materials, these devices suffer from low current density, charge injection imbalance and exciton ionization caused by large applied bias voltages ${ }^{12}$. Recently, an infrared quantum-dot LED based on direct exciton generation through carrier injection achieved $1.15 \%$ external quantum efficiency (EQE), but the organic carrier-injection layer limited the current density and, as a result, the radiance ${ }^{7}$ $\left(0.0067 \mathrm{~W} \mathrm{sr}^{-1} \mathrm{~m}^{-2}\right)$. So far, the brightest infrared quantum-dot
LEDs have achieved a radiance of $0.8 \mathrm{~W} \mathrm{sr}^{-1} \mathrm{~m}^{-2}$ (see Supplementary Section SA), with $0.5 \%$ EQE (ref. 4). In visiblewavelength quantum-dot LEDs, inorganic charge-transport layers ( $\mathrm{ZnO}: \mathrm{SnO}_{2}$ alloy for electrons and $\mathrm{NiO}$ for holes) have recently been used to increase the current density to a few amperes per square centimetre, with a consequent significant improvement in radiance $^{6}$. These results directly reflect the improved performance of the charge-injection layers of such devices.

Charge-carrier dynamics in the quantum-dot layer also play a critical role in device performance. Increasing the carrier injection rate while avoiding excessive non-radiative decay through dissociation or field ionization and eventual trapping of the charge carriers requires a delicate balance of the relevant physical processes. The requirements for successful operation of quantum-dot LEDs are more constrained than those for the operation of quantumdot photovoltaic devices. As well as overcoming the challenge of achieving efficient inter-dot charge transfer, which is common to both devices, an LED must also balance charge injection against efficient radiative recombination within the quantum-dot layer. The competition between exciton dissociation and radiative recombination can be controlled by tailoring the inter-dot separation using linker molecules of different lengths ${ }^{13}$. The crucial development we report here is the ability to dramatically enhance the quantum efficiency of exciton recombination by tuning the distance between the $\mathrm{PbS}$ quantum dots in the active layer of an LED. For linker molecules with between three and eight $\mathrm{CH}_{2}$ groups, the quantum efficiency can vary by over two orders of magnitude. By fabricating devices with optimal linker length as well as high-performance carrier-injection layers, we demonstrate infrared quantum-dot LEDs with an eightfold increase in radiance and twofold increase in EQE compared with previous best devices.

The schematic structure of the quantum-dot LEDs is shown in Fig. 1a, and the rationale for their operation can be understood from the relevant energy levels in Fig. 1b. Lead-salt quantum dots are well established as efficient emitters in the near-infrared, and in this work we focus on $\mathrm{PbS}$ quantum dots because their absolute energy levels ${ }^{14}$ are most appropriate (Fig. 1b). The $\mathrm{ZnO}$ layer is intended to inject electrons into the quantum dots and block holes, and the poly(3,4-ethylenedioxythiophene) poly(styrenesulphonate) (PEDOT:PSS) film should inject holes and block electrons. We fabricated the electron-injecting layers using colloidal $\mathrm{ZnO}$ nanoparticles for several reasons. First, the electron mobility of $\mathrm{ZnO}$ (ref. 15) is about two orders of magnitude higher than that of the commonly used tris(8-hydroxyquinolinato) aluminium $(\mathrm{Alq} 3)^{16}$. Second, the $\mathrm{ZnO}$ prevents direct contact between the

'School of Applied and Engineering Physics, Cornell University, Ithaca, New York 14853, USA, ${ }^{2}$ School of Chemical and Biomolecular Engineering, Cornell University, Ithaca, New York 14853, USA, ${ }^{3}$ Department of Materials Science and Engineering, Cornell University, Ithaca, New York 14853, USA; "Present address: Department of Physics and Astronomy, Center for Photochemical Sciences, Bowling Green State University, Bowling Green, Ohio 43403, USA (L.S.); Department of Bioelectronics, Ecole Nationale Supérieure des Mines, CMP-EMSE, MOC, Gardanne, France (G.G.M.); ;These authors contributed equally to this work. *e-mail: Is462@cornell.edu; fww1@cornell.edu 

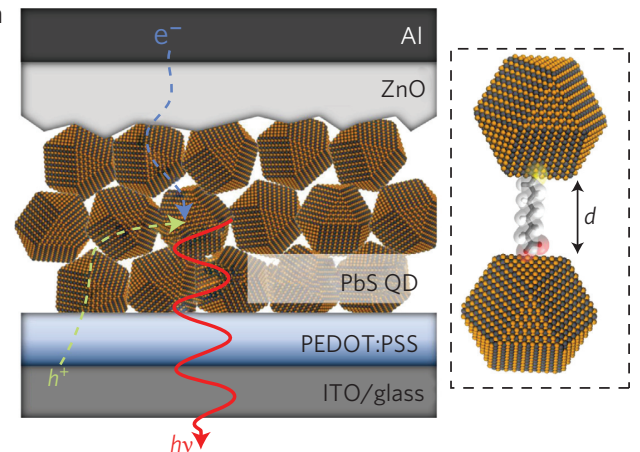

b Vacuum

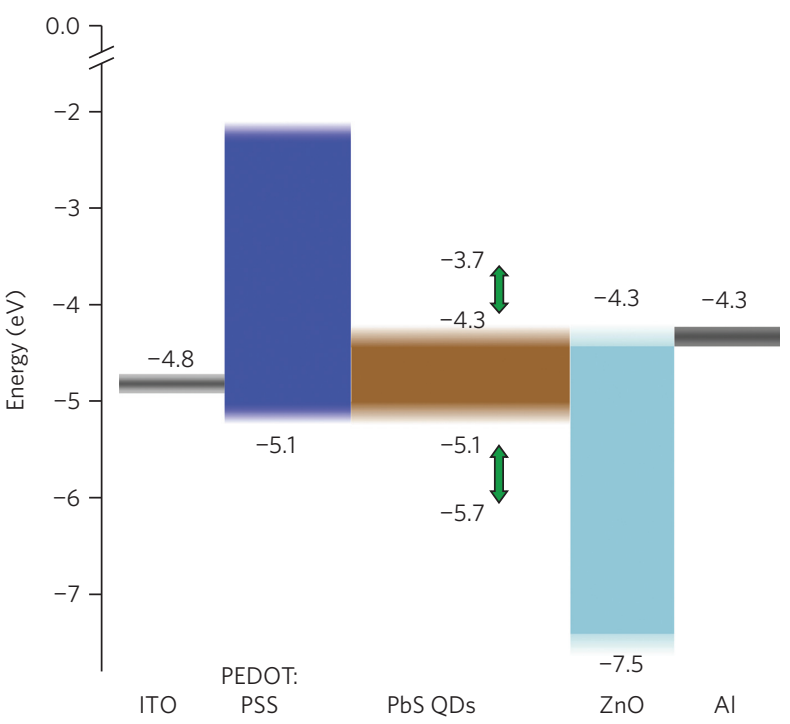

Figure 1 | Physical and electronic structure of the LEDs. a, Schematic device structure. b. Electronic energy levels of each material in the device stack. Energies are relative to vacuum, which is defined as the zero of electron energy. Arrows indicate the tuning range of the electron affinity (from $-3.7 \mathrm{eV}$ to $-4.3 \mathrm{eV}$ ) and the ionization potential (from $-5.7 \mathrm{eV}$ to $-5.1 \mathrm{eV}$ ) by changing the diameter (from $2.7 \mathrm{~nm}$ to $6.5 \mathrm{~nm}$ ) of the quantum dots.

quantum dots and the aluminium electrode, thereby avoiding plasmonic quenching of the quantum-dot emission ${ }^{17}$. Finally, the use of colloidal $\mathrm{ZnO}$ nanoparticles avoids damage to the quantum dots, which would probably occur with $\mathrm{ZnO}$ layers deposited by sputtering $^{18}$. The transparent conductor PEDOT:PSS was chosen as a high-conductivity $\left(\sim 1 \times 10^{-3} \mathrm{~S} \mathrm{~cm}^{-1}\right)$ hole-transporting contact to the quantum-dot film. Nearly ohmic contacts are formed between the quantum-dot layer and the carrier-transporting layers due to the close energy-level alignment.

Colloidal $\mathrm{PbS}$ quantum dots were synthesized using organometallic precursors ${ }^{19,20}$. We successively spin coated the PEDOT:PSS, $\mathrm{PbS}$ quantum dots and $\mathrm{ZnO}$ nanoparticle layers onto cleaned, prepatterned indium tin oxide (ITO) substrates. To couple the quantum dots within the active layer, the long-chain oleate ligands were displaced by bifunctional linker molecules of controlled length. Specifically, we used mercapto alkyl carboxylic acids of variable alkyl chain lengths, including 3-mercaptopropionic acid (MPA), 6-mercaptohexanoic acid (MHA), 8-mercaptooctanoic acid (MOA) and 11-mercaptoundecanoic acid (MUA). The active layer was formed by repeated cycles of quantum-dot spin-casting and linker treatment, providing robust, crack-free films ${ }^{10}$. The aluminium top electrode was deposited by thermal evaporation on the $\mathrm{ZnO}$.

Small separation favours efficient charge transport (in the plane of the quantum-dot layer as well as in the injection direction), but
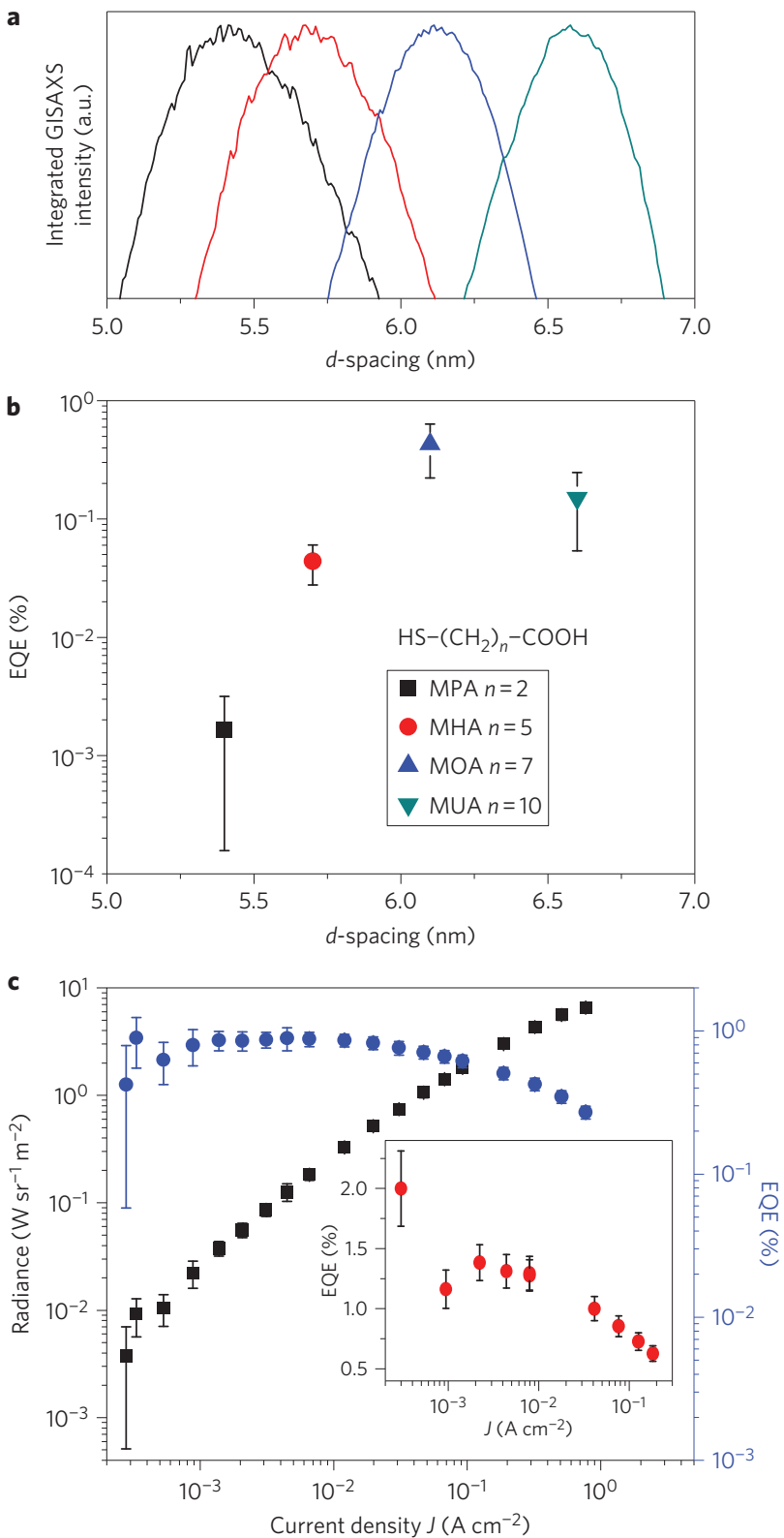

Figure 2 | Relationship between LED performance and inter-dot distance. a, Azimuthally integrated GISAXS intensity of quantum-dot films with varying linker length (see Supplementary Section SE). b, EQE dependence on inter-dot distance. Error bars indicate EQE fluctuations in a broad range of bias ( 0.7-11 V) and from diode to diode. c, Dependence of radiance (black squares) and EQE (blue circles) on current density for the device with maximum radiance. Inset: dependence of EQE on current density for the device with maximum EQE. Error bars indicate overall experimental errors (see Supplementary Section SB). All measurements were taken from devices made using quantum dots with diameters of $4.5 \mathrm{~nm}$, except the inset, which was made using quantum dots with diameters of $3.5 \mathrm{~nm}$.

also leads to more rapid exciton dissociation ${ }^{13}$. On the other hand, larger spacing promotes radiative recombination, but charge injection is more difficult. We determined the distance between the quantum dots in the films using grazing-incidence small-angle X-ray scattering (GISAXS) (Fig. 2a). The electroluminescence quantum efficiencies of the films were measured (see Methods) as a function of the inter-dot distance.

When the inter-dot spacing increased from 5.4 to $6.1 \mathrm{~nm}$, the EQE increased by a factor of $\sim 150$ (Fig. $2 b$ ). With a further increase 
in inter-dot distance, the efficiency declined. The measured photoluminescence lifetimes and quantum efficiencies of the same quantum-dot films increased monotonically and by a factor of $\sim 20$ with inter-dot distance (see Supplementary Section SJ). However, increasing the spacing between quantum dots also weakened the screening of any external field. This in turn increased the probability of exciton ionization ${ }^{12}$, which effectively decreases electroluminescence efficiency. The maximum EQE was obtained with MOA for all quantum-dot diameters. We also measured the $\mathrm{EQE}$ as a function of current density for each linker. For most devices the EQE increased slightly, then decreased, with current density (Fig. 2c). The EQE varied by less than a factor of 10 , whereas the current density varied over two to three orders of magnitude. The current density at which the EQE reaches its maximum value depends on the linker molecule (see Supplementary Section $\mathrm{SF}$ ). Finally, the EQE depends on the size of the quantum dots, even for a fixed linker. Although the measured EQE fluctuated from device to device, the overall trend was that the EQE decreased as quantum-dot size increased (see Supplementary Section SD), which is consistent with the trend for photoluminescence quantum efficiency ${ }^{21}$. The highest EQE $(2.0 \pm 0.3 \%)$ was obtained from MOA-capped quantum dots, with an emission peak at $1,054 \mathrm{~nm}$ (Fig. 2c, inset). With $1.2 \mathrm{~V}$ applied bias, the electroluminescence power was $185 \mathrm{nW}$. The corresponding internal quantum efficiency was estimated to be $8 \%$, based on a calculation that assumes Lambertian emission (see Methods). The highest radiance was obtained from a device with MOA-capped quantum dots (diameter, $4.5 \mathrm{~nm}$ ) with an emission peak at 1,232 nm (Fig. 2c). This device emitted $60 \mu \mathrm{W}$ from a pixel of area $0.03 \mathrm{~cm}^{2}$, which corresponds to a radiance of $6.4 \mathrm{~W} \mathrm{sr}^{-1} \mathrm{~m}^{-2}$. This value is eight times greater than the highest radiance of a previously reported infrared quantum-dot $\mathrm{LED}^{4}$. The electroluminescence power is comparable to state-of-the-art infrared LEDs (for example, commercial InGaAsP LEDs) fabricated by planar epitaxial technology over the range $900-1,300 \mathrm{~nm}$.

We believe that the competition between exciton dissociation (and eventual non-radiative recombination) and radiative recombination dominates the variation of EQE with the different linker molecules. However, other effects may play a role. Changing the linker molecules may change the passivation of the quantum-dot surfaces to some extent, in addition to changing the electronic coupling between quantum dots. The balance of charge injection and the

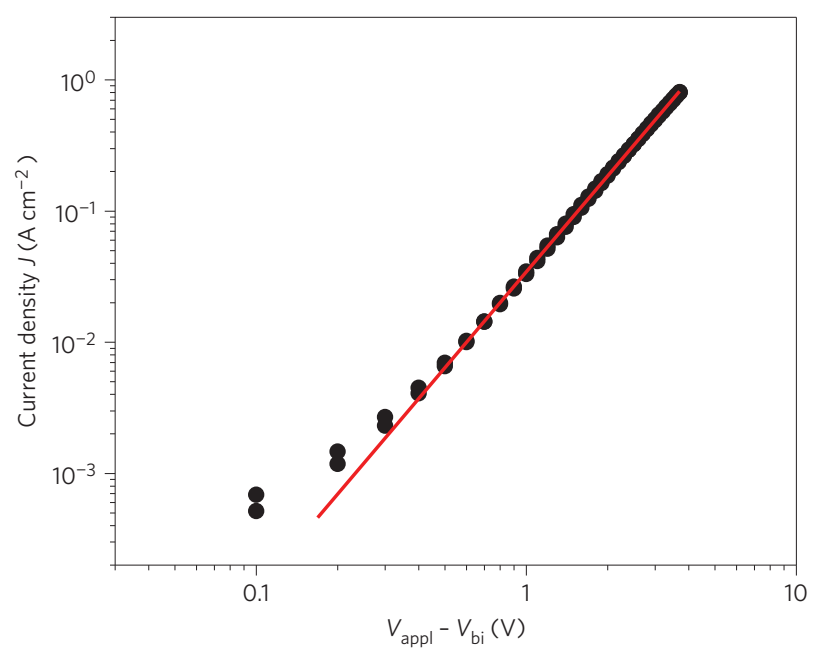

Figure 3 | Current density-voltage characteristic of a device made of MOA-capped quantum dots with diameters of $4.5 \mathrm{~nm}$. The slope of the solid line is 2.1. Scans acquired with increasing and decreasing bias are displayed, and slight hysteresis is observed.

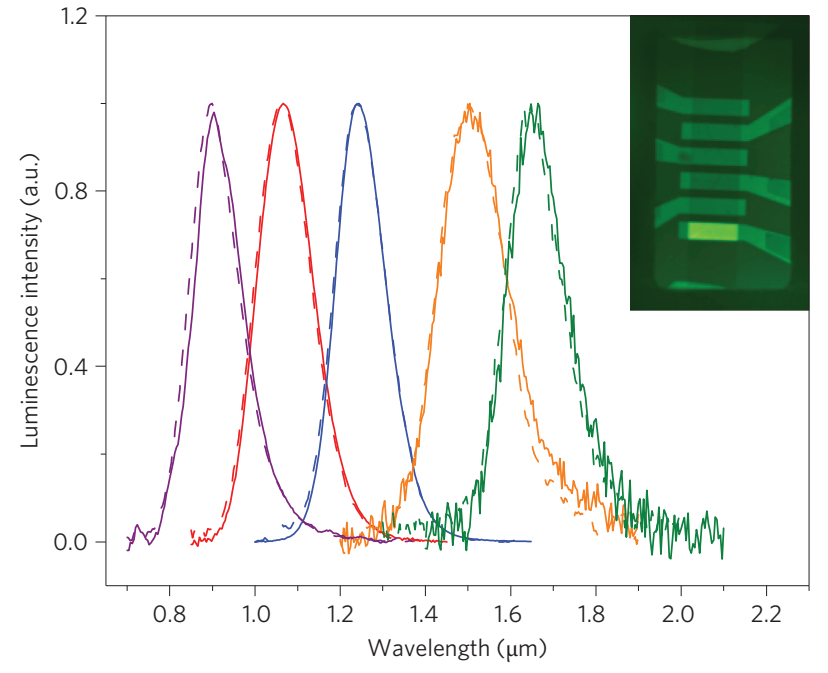

Figure 4 | Emission spectra and infrared image of LEDs. Normalized electroluminescence (solid) and photoluminescence (dashed) spectra of LEDs made from different sized quantum dots (from left to right: $2.7 \mathrm{~nm}$, $3.5 \mathrm{~nm}, 4.5 \mathrm{~nm}, 5.6 \mathrm{~nm}$ and $6.5 \mathrm{~nm}$ in diameter), all capped by MHA linkers. Inset: photograph of a device emitting at 1,244 $\mathrm{nm}$ taken by an InGaAs camera.

efficiency of coupling out emitted light will also be influenced by the choice of linker molecules. Further work will be needed to quantitatively assess the role of these processes. Finally, we estimate that the number of excitons per quantum dot is always well below one. Thus, Auger relaxation of multiple excitons plays a negligible role in the dynamics.

The quantum-dot LEDs show good rectification behaviour (see Supplementary Section SC). A representative current-voltage characteristic (corrected for the built-in potential ${ }^{22}$ ) reveals a single operation regime with a slope of 2.1 (Fig. 3), which implies that the current is space-charge-limited. The high mobility of the carrier-transporting materials facilitates a high current density $\left(\sim 1 \mathrm{~A} \mathrm{~cm}^{-2}\right)$ at a fairly low bias $(\sim 4.5 \mathrm{~V})$ in these devices (Fig. 3). The current density is improved by several orders of magnitude compared with previous quantum-dot LEDs ${ }^{4,5,7,8}$, and this contributes to the high radiance of our devices.

Figure 4 presents electroluminescence and photoluminescence spectra of quantum-dot LEDs with different sizes of quantum dots. The emission peak can be tuned from $950 \mathrm{~nm}$ up to $1,650 \mathrm{~nm}$, and the emission tail extends from $800 \mathrm{~nm}$ to beyond $1,850 \mathrm{~nm}$. There is virtually no broadening or shift of the electroluminescence spectra with respect to the photoluminescence spectra. The radiance is at a maximum for devices that emit between 900 and $1,300 \mathrm{~nm}$, and decreases outside that range, while the current is approximately constant. The infrared image of a typical device (Fig. 4, inset) shows uniform emission near $1,244 \mathrm{~nm}$, which is evidence of uniform carrier transport and quantum-dot active layers.

The performance of the quantum-dot LEDs presented here can compete with state-of-the-art infrared LEDs fabricated by planar epitaxy. Moreover, we are optimistic that further performance improvements are possible based on our understanding of the limitations of charge transport and injection into the quantum-dot active layer. In general, fine-tuning of the mobilities and carrier-injection rates can optimize LED efficiency and radiance ${ }^{23}$. The PbS quantum dots capped by mercapto alkyl carboxylic acid linkers are effectively p-type ${ }^{24}$; the electron mobility in the film is lower than the hole mobility and, as a result, electron-hole recombination may occur near the cathode ${ }^{23}$. This non-uniform distribution of recombination 
may limit device efficiency. The mobilities can be balanced by treating the quantum dots with amine-based linker molecules, as has been done for PbSe (refs 25,26) quantum dots. On the other hand, the difference of the conductivities of the electron- and hole-transporting layers causes imbalance in the carrier injection, which consequently charges the quantum dots. Charging increases the probability of non-radiative Auger relaxation, which quenches the luminescence ${ }^{17}$. However, this imbalance can be tuned by adjusting the conductivity of the PEDOT:PSS film by changing the ratio of PSS to $\mathrm{PEDOT}^{1}$, or the $\mathrm{ZnO}$ film through photodoping (see Supplementary Section SH).

By controlling the distance between quantum dots on the ångstrőm level, exciton recombination in a quantum-dot film can be enhanced dramatically, and this leads to an order-of-magnitude improvement in infrared LEDs. Further improvements can be expected as the surface passivation of lead salts and other infrared nanostructures is improved. These solution-processed emitters may be integrated easily with other materials. The results and approach presented here could be extended to a broad range of nanostructure-based optoelectronic devices.

\section{Methods}

LED device fabrication. Pre-patterned ITO-coated glass substrates (Kintec) were cleaned and treated with UV-ozone for 10 min. PEDOT:PSS (product no. AI4083, H.C. Starck) was filtered through a $0.45 \mu \mathrm{m}$ polyvinylidene fluoride (PVDF) syringe filter and spin-cast onto the cleaned ITO substrate at 6,000 r.p.m. for $1 \mathrm{~min}$ then baked on a hot plate at $170{ }^{\circ} \mathrm{C}$ for $4 \mathrm{~min}$. $\mathrm{PbS}$ quantum dots were spin-cast from a $30 \mathrm{mg} \mathrm{ml}^{-1}$ chlorobenzene solution at 1,000 r.p.m. for $30 \mathrm{~s}$. The quantum-dot film was then treated with a 1:9 (by volume) solution of MXA $(\mathrm{X}=\mathrm{P}, \mathrm{H}, \mathrm{O})$ in acetonitrile (for the MUA solution, powder MUA was dissolved in acetonitrile at room temperature to saturation and filtered through a $0.2 \mu \mathrm{m}$ PVDF syringe filter) and rinsed with pure acetonitrile and chlorobenzene by dispensing the solution on top of the film and spin-casting at 1,000 r.p.m. for 30 s. The rinsing procedure was repeated six times to ensure complete removal of free ligand molecules. This protocol constituted one cycle of quantum-dot layer deposition. For all devices, three cycles of deposition were performed. After deposition of the quantum-dot film, $20 \mathrm{mg} \mathrm{ml}^{-1}$ of a $\mathrm{ZnO}$ nanoparticle solution was spin-cast at 1,000 r.p.m. for $1 \mathrm{~min}$. Three such depositions of $\mathrm{ZnO}$ nanoparticles were carried out to ensure complete coverage. Following $\mathrm{ZnO}$ deposition, 600 Å of aluminium was deposited by thermal evaporation in vacuum $\left(\sim 1 \times 10^{-6}\right.$ torr). The entire device fabrication sequence, except for the metal evaporation step, was performed in ambient air. Each $25 \mathrm{~mm} \times$ $25 \mathrm{~mm}$ substrate was patterned to yield six devices, each with an area of $0.03 \mathrm{~cm}^{2}$

LED characterization. Current-voltage characteristics were recorded using a computer-controlled Keithley 236 source measurement unit. To calculate the EQEs, electroluminescence from the front face of the device was detected using a calibrated Newport 918D-IR-OD3 germanium photodetector at the same time that the $J-V$ characteristics were measured. Lambertian emission was assumed. The correction factor was calculated according to the detector position relative to the LED and the size of the active area of the detector (solid angle subtended by the detector). As verification, we used a NIST-traceable integrating sphere to measure the electroluminescence power from a quantum-dot LED that emits near $900 \mathrm{~nm}$. We found the electroluminescence powers obtained by each method were the same.

Electroluminescence spectra were measured with a Princeton Instruments SP2300 monochromator and infrared detectors (including an infrared femtowatt photoreceiver, New Focus 2153, and a TE-cooled InGaAs detector from Judson Technologies) with bias applied to the device using a SourceMeter instrument (Keithley 2400). Photoluminescence spectra were measured in the same experimental set-up, with a green laser (wavelength, $532 \mathrm{~nm} ; \mu$ Flare OEM laser system, Lumanova) as the excitation source instead of applying a bias to the device.

Calculation of EQE AND IQE. The EQE was calculated by dividing the number of emitted photons (calculated from electroluminescence power and wavelength) by the number of injected electron-hole pairs (calculated from the current). The IQE was calculated using the relation ${ }^{27} \mathrm{IQE}=2 n^{2} \mathrm{EQE}$, where $n$ is the refractive index of the materials between the emitters and air. We chose the refractive index of glass (1.45) for $n$ because glass comprises the largest volume of a quantum-dot LED. Thus, the conversion factor from EQE to IQE was calculated to be 4. Alternatively, we could estimate the conversion factor to be in the range 3-6 by following ref. 3 , in which the quantum-dot LEDs have the same structure at the light exit side (PEDOD:PSS/ITO/glass). This estimate takes into account the effects of the PEDOT:PSS and ITO layers.

X-ray scattering characterization. The samples for GISAXS measurements were prepared by spin-coating a PEDOT:PSS layer on top of a cleaned silicon wafer at
6,000 r.p.m. for $1 \mathrm{~min}$. After baking the PEDOT:PSS film at $170{ }^{\circ} \mathrm{C}$ for $4 \mathrm{~min}$, quantum-dot films with variable-length linkers were deposited in a manner identical to the LED device film preparation. The GISAXS measurements were performed on beamline D1 of the Cornell High Energy Synchrotron Source (CHESS) using monochromatic radiation of wavelength $\lambda=1.264 \AA$ with a bandwidth $\Delta \lambda / \lambda$ of $1.5 \%$. The X-ray beam was produced by a hardbent dipole magnet of the Cornell storage ring and monochromatized with $\mathrm{Mo}: \mathrm{B}_{4} \mathrm{C}$ synthetic multilayers with a period of $30 \AA$. The D1 area detector (MedOptics) is a fibre-coupled charge-coupled device camera with a pixel size of $46.9 \mu \mathrm{m} \times 46.9 \mu \mathrm{m}$ and a total of $1,024 \times 1,024$ pixels with a 14-bit dynamical range per pixel. Typical readout time per image was less than $5 \mathrm{~s}$. The images were dark-current-corrected, distortion-corrected and flat-field-corrected by the acquisition software. The sample-to-detector distance was $910.5 \mathrm{~mm}$, as determined using a silver behenate powder standard. The incident angle of the X-ray beam was $0.25^{\circ}$, that is, slightly above the silicon critical angle. Typical exposure times ranged from 0.1 to $3.0 \mathrm{~s}$. Scattering images were calibrated and integrated using Fit2D software.

\section{Received 28 December 2011; accepted 30 March 2012;} published online 6 May 2012

\section{References}

1. Cho, K. et al. High-performance crosslinked colloidal quantum-dot light-emitting diodes. Nature Photon. 3, 341-345 (2009).

2. Colvin, V. L., Schlamp, M. C. \& Alivisatos, A. P. Light-emitting diodes made from cadmium selenide nanocrystals and a semiconducting polymer. Nature 370, 354-357 (1994).

3. Coe, S., Woo, W., Bawendi, M. \& Bulović, V. Electroluminescence from single monolayers of nanocrystals in molecular organic devices. Nature 420, 800-803 (2002)

4. Tessler, N., Medvedev, V., Kazes, M., Kan, S. \& Banin, U. Efficient near-infrared polymer nanocrystal light-emitting diodes. Science 295, 1506-1508 (2002).

5. Steckel, J. S., Coe-Sullivan, S., Bulović, V. \& Bawendi, M. G. $1.3 \mu \mathrm{m}$ to $1.55 \mu \mathrm{m}$ tunable electroluminescence from $\mathrm{PbSe}$ quantum dots embedded within an organic device. Adv. Mater. 15, 1862-1866 (2003).

6. Caruge, J. M., Halpert, J. E., Wood, V., Bulović, V. \& Bawendi, M. G. Colloidal quantum-dot light-emitting diodes with metal-oxide charge transport layers. Nature Photon. 2, 247-250 (2008).

7. Bourdakos, K. N., Dissanayake, D. M. N. M., Lutz, T., Silva, S. R. P. \& Curry, R. J. Highly efficient near-infrared hybrid organic-inorganic nanocrystal electroluminescence device. Appl. Phys. Lett. 92, 153311 (2008).

8. Konstantatos, G., Huang, C., Levina, L., Lu, Z. \& Sargent, E. H. Efficient infrared electroluminescent devices using solution-processed colloidal quantum dots. Adv. Funct. Mater. 15, 1865-1869 (2005).

9. Wise, F. W. Lead salt quantum dots: the limit of strong quantum confinement Acc. Chem. Res. 33, 773-780 (2000).

10. Choi, J. J. et al. PbSe nanocrystal excitonic solar cells. Nano Lett. 9, 3749-3755 (2009).

11. Konstantatos, G. et al. Ultrasensitive solution-cast quantum dot photodetectors Nature 442, 180-183 (2006).

12. Huang, H., Dorn, A., Nair, G. P., Bulović, V. \& Bawendi, M. G. Bias-induced photoluminescence quenching of single colloidal quantum dots embedded in organic semiconductors. Nano Lett. 7, 3781-3786 (2007).

13. Choi, J. J. et al. Photogenerated exciton dissociation in highly coupled lead salt nanocrystal assemblies. Nano Lett. 10, 1805-1811 (2010).

14. Hyun, B. R. et al. Electron injection from colloidal PbS quantum dots into titanium dioxide nanoparticles. ACS Nano 2, 2206-2212 (2008).

15. Verbakel, F., Meskers, S. C. J. \& Janssen, R. A. J. Electronic memory effects in diodes of zinc oxide nanoparticles in a matrix of polystyrene or poly(3hexylthiophene). J. Appl. Phys. 102, 083701 (2007).

16. Kepler, R. G. et al. Electron and hole mobility in tris(8-hydroxyquinolinolatoN1,O8) aluminum. Appl. Phys. Lett. 66, 3618-3620 (1995).

17. Shimizu, K. T., Woo, W. K., Fisher, B. R., Eisler, H. J. \& Bawendi, M. G. Surfaceenhanced emission from single semiconductor nanocrystals. Phys. Rev. Lett. 89, 117401 (2002).

18. Barkhouse, D. A., Kramer, I. J., Wang, X. \& Sargent, E. H. Dead zones in colloidal quantum dot photovoltaics: evidence and implications. Opt. Express $\mathbf{1 8}$, A451-A457 (2010).

19. Murray, C. B., Kagan, C. R. \& Bawendi, M. G. Synthesis and characterization of monodisperse nanocrystals and close-packed nanocrystal assemblies. Annu. Rev. Mater. Sci. 30, 545-610 (2000).

20. Hines, M. A. \& Scholes, G. D. Colloidal PbS nanocrystals with size-tunable nearinfrared emission: observation of post-synthesis self-narrowing of the particle size distribution. Adv. Mater. 15, 1844-1849 (2003).

21. Semonin, O. E. et al. Absolute photoluminescence quantum yields of IR-26 dye, PbS, and PbSe quantum dots. J. Phys. Chem. Lett. 1, 2445-2450 (2010).

22. Malliaras, G. G., Salem, J. R., Brock, P. J. \& Scott, C. Electrical characteristics and efficiency of single-layer organic light-emitting diodes. Phys. Rev. B 58, R13411-R13414 (1998). 
23. Malliaras, G. G. \& Scott, J. C. The roles of injection and mobility in organic light emitting diodes. J. Appl. Phys. 83, 5399-5403 (1998).

24. Pattantyus-Abraham, A. G. et al. Depleted-heterojunction colloidal quantum dot solar cells. ACS Nano 4, 3374-3380 (2010).

25. Talapin, D. V. \& Murray, C. B. PbSe nanocrystal solids for $\mathrm{n}$ - and $\mathrm{p}$-channel thin film field-effect transistors. Science 310, 86-89 (2005).

26. Law, M. et al. Structural, optical, and electrical properties of PbSe nanocrystal solids treated thermally or with simple amines. J. Am. Chem. Soc. 130, 5974-5985 (2008).

27. Greenham, N. C., Friend, R. H. \& Bradley, D. D. C. Angular dependence of the emission from a conjugated polymer light-emitting diode: implications for efficiency calculations. Adv. Mater. 6, 491-494 (1994).

\section{Acknowledgements}

This material is based on work supported by the National Science Foundation (NSF, grant no. EEC-0646547) and by the New York State Foundation for Science, Technology and Innovation (NYSTAR). J.J.C. and D.S. acknowledge support from the Cornell Center for Materials Research with funding from IGERT: a Graduate Traineeship in Nanoscale

Control of Surfaces and Interfaces (DGE-0654193) of the NSF. This publication is based on work supported in part by an award (no. KUS-C1-018-02) made by King Abdullah University of Science and Technology (KAUST). GISAXS measurements were conducted at Cornell High Energy Synchrotron Source (CHESS) and the authors thank D.-M. Smilgies for calibration of the beam line set-up.

\section{Author contributions}

L.S. and J.J.C. conceived and designed the experiments. L.S. and D.S. performed device characterization and optical measurements. J.J.C. synthesized the materials, fabricated the devices, and performed GISAXS and optical measurements. A.C.B. calculated the energy levels of the quantum dots. L.S. and F.W.W. co-wrote the paper. F.W.W., T.H. and G.G.M. (now at Ecole Nationale Supérieure des Mines, France) supervised the project. All authors discussed the work, commented on the manuscript and contributed to revision of the manuscript.

\section{Additional information}

The authors declare no competing financial interests. Supplementary information accompanies this paper at www.nature.com/naturenanotechnology. Reprints and permission information is available online at http://www.nature.com/reprints. Correspondence and requests for materials should be addressed to L.S. and F.W.W. 